\title{
Numerical Investigation of Optimal Dynamic Measurements
}

\author{
Aleksandr L. Shestakov, Georgy A. Sviridyuk, Alevtina V. Keller, Alyona A. Zamyshlyaeva, Yurii V. \\ Khudyakov
}

South Ural State University, Lenina, 76, Chelyabinsk, Russia

\section{ABSTRACT}

The basic ideas of mathematical modeling of optimal dynamic measurements are presented. The key thing is to construct a mathematical model of the measuring transducer, which allows simulating also a complex measuring transducer. In this manuscript we propose such a mathematical model and we discuss its results obtained through numerical studies in a variety of cases: from the simplest, without considering disturbances, to the case when a deterministic disturbance, for example resonances, occur at the input and at the output of the measuring transducer. Lastly, the importance of the description of the set of admissible measurements in the modeling of dynamic measurements is discussed.

\section{Section: RESEARCH PAPER}

Keywords: mathematical model of measuring transducer; optimal dynamic measurement; set of admissible measurements

Citation: Aleksandr L. Shestakov, Georgy A. Sviridyuk, Alevtina V. Keller, Alyona A. Zamyshlyaeva, Yurii V. Khudyakov, Numerical Investigation of Optimal Dynamic Measurements, Acta IMEKO, vol. 7, no. 2, article 12, June 2018, identifier: IMEKO-ACTA-07 (2018)-02-12

Section Editor: Franco Pavese, Italy

Received December 16, 2017; In final form May 11, 2018; Published June 2018

Copyright: (c) 2018 IMEKO. This is an open-access article distributed under the terms of the Creative Commons Attribution 3.0 License, which permits unrestricted use, distribution, and reproduction in any medium, provided the original author and source are credited

Funding: The work was supported by Act 211 Government of the Russian Federation, contract № 02.A03.21.0011

Corresponding author: Alevtina Keller, e-mail: kellerav@susu.ru

\section{INTRODUCTION}

Currently, the theory and practice of dynamic measurements are developing in various directions. For a long time the theory of inverse problems [1], methods of direct and inverse Fourier transforms were the mathematical basis of dynamic measurements. In addition, for more than 20 years methods of the remote control theory were successfully applied for solving various problems of dynamic measurements. In the recent years, a new approach was developed [2] to restore the input signal distorted by the measuring transducer and external interference, called by the authors "the theory of optimal dynamic measurements". We emphasize that the theory of optimal dynamic measurements is based on three fundamental components: 1) the solution of dynamic measurements problems using the ideas and methods of the remote control theory; 2) the development of the descriptor systems theory and its applications [3]; 3) the development of methods for the solution of Sobolev type equations and corresponding optimal control problems, including numerical methods for solving optimal control problems for Leontief type systems. Leontief type systems are finite-dimensional case of Sobolev-type equations and a special case of descriptor systems (with constant matrices). This report presents recent results in the theory of optimal dynamic measurements.

\section{MATHEMATICAL MODELING OF OPTIMAL DYNAMIC MEASUREMENTS}

We first introduce mathematical model of optimal dynamic measurements (Figure 1).

Mathematical model of measuring transducer (MT) is represented by the Leontief type system of equations

$\left\{\begin{array}{c}L \dot{x}=A x+B u+G \varsigma, \\ y=C x+D \eta,\end{array}\right.$

where $L$ and $A$ are matrices that characterize the structure of the MT. In some cases it is possible that $\operatorname{det} L=0$ (this will be discussed in the next section); $x(t)$ and $\dot{x}(t)$ are vector functions of the state of the MT and the velocity of the state change, respectively; $y(t)$ is a vector-function of observation; $C$ and $D$ are rectangular matrices characterizing the interrelation between the system state and observation; $u(t)$ is a vectorfunction of measurements; $B$ is the matrix characterizing the 


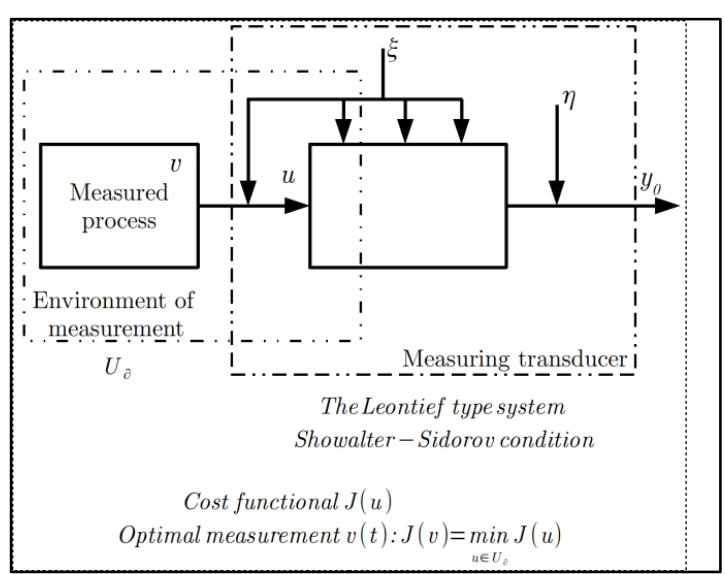

Figure 1. Structure elements of mathematical model of optimal dynamic measurements.

interrelation between the system state and measurement; $\varsigma(t)$ is a vector-function of resonances in the circuits of the MT (see Figure 1); $G$ is the matrix characterizing influence of resonances in the circuits of the MT; $\eta(t)$ is a vector-function of disturbance at the output of the the MT (see Figure 1). If $L$ is not degenerate then system (1) can be reduced to

$\left\{\begin{array}{c}\dot{x}=M x+F u+H \varsigma, \\ y=C x+D \eta,\end{array}\right.$

where $M=L^{-1} A, F=L^{-1} B, H=L^{-1} G$.

This system appeared in the dynamic measurements theory from the remote control theory, where (2) was obtained in the study of the transfer function of the MT. Finally, in accordance to new approaches in the field of measurements, it is possible to replace the terms "input signal" with "measurement" and "output signal" with "observation" [4], respectively. We emphasize that all observations and measurements in systems (1) and (2) are simulated or "virtual".

The initial Showalter - Sidorov condition

$$
\left[(\alpha L-A)^{-1} L\right]^{p+1}\left(x(0)-x_{0}\right)=0
$$

for some $x_{0} \in R^{n}, \alpha \in \rho^{L}(M)$, reflects the initial state of the MT. The initial Showalter - Sidorov condition is equivalent to the initial Cauchy condition $x(0)=x_{0}$ when $\operatorname{det} L \neq 0$.

For the investigation of problem (1), (3) introduce the space of states $X=\left\{x \in L_{2}\left((0, \tau), R^{n}\right): \dot{x} \in L_{2}\left((0, \tau), R^{n}\right)\right\}$ and the space of observations $Y=C[X]$. The key concept is the optimal measurement simulated by solving the optimal control problem for (1), (3). To find it, we introduce the space of measurements $U=\left\{u \in L_{2}\left((0, \tau), R^{n}\right): u^{(p+1)} \in L_{2}\left((0, \tau), R^{n}\right)\right\}$ and allocate it in a closed convex set of admissible measurements $U_{\partial} \subset U$

$U_{\partial}=\left\{u \in U: \sum_{q=0}^{\theta} \int_{0}^{\tau}\left\|u^{(q)}(t)\right\|^{2} d t \leq d\right\}$.

The latter contains a priori information about measurements (this will be discussed in the sixth section of the paper). In the optimal measurements theory, as in the control theory, several types of cost functionals can be used depending on the goals of restoration of a dynamically distorted signal.

Consider a general cost functional

$J(u)=J_{1}\left(y(u, \varsigma, \eta, t)-y_{0}(t)\right)+J_{2}(u, \varsigma)$,

where $y_{0}(t), t \in[0, \tau]$ is a continuously differentiable function constructed on the basis of the observed values $Y_{0 i}$ at the output of the real MT, which is called "real observation" in the mathematcal model.

The first term of the cost functional reflects the closeness of the real observation $y_{0}(t)$ and the virtual observation $y(t)$, obtained on the basis of mathematical model of the MT. The second term acts as a filter in the presence of resonances $\varsigma(t)$ in the chains of the MT. In case of lack of resonances $s(t)$ in the chains of the MT, functional (5) consists only of the first term.

The minimum point $v(t)$ of the functional (5) on the set $U_{\partial}$, being a solution of optimal control problem

$J(v)=\min _{u \in U_{\partial}} J(u)$

is called an optimal dynamic measurement. In practice, there is only indirect information about $v(t)$.

Note that quadratic functionals containing the observational error and its derivative have also been considered in the remote control theory, moreover issues of stability arising in connection with this were actively investigated. One of the first works that used the criterion of quadratic error is the article by A. A. Kharkevich [5]. It describes methods for estimating distortions introduced by linear systems. In the $50 \mathrm{~s}$ the integral criteria of quadratic error began to be used: 1) to search the optimal parameters of the system modeling the dynamics [6], [7] and 2) to solve the problem of synthesis of the regulator [8]. It should be noted that numerical methods for the calculation of the real automatic systems were developed by $\mathrm{K}$. Merriam [9].

Solutions were also made by working in the frequency domain and by using the spectra of disturbances and formulas for squared deviations, which allowed to reduce the problem of finding the best operator to the search of a function, delivering an extremum to the mean-square functional [10].

\section{MATHEMATICAL MODEL OF A COMPLEX MEASURING TRANSDUCER AS A LEONTIEF TYPE SYSTEM}

The mathematical model of a complex measuring transducer involves several subsystems, each of which represents a model of one measuring transducer (or simple MT). Differential equations in the system reflect a complex of dynamic system elements (subsystems), and algebraic equations represent the connection between dynamic elements.

Theoretical aspects of the construction of mathematical models of the complex MT are based on the ideas of the descriptor systems theory [11]. As an example, we present the mathematical model of the complex MT, using the iterative principle for the restoration of a dynamically distorted signal, which allows to achieve a high dynamic accuracy during dynamic measurements [12].

A structural scheme of the iterative MT is shown in Figure 2 for the case $\mathrm{N}=3$.

The mathematical model of each simple transducer is represented by system: 


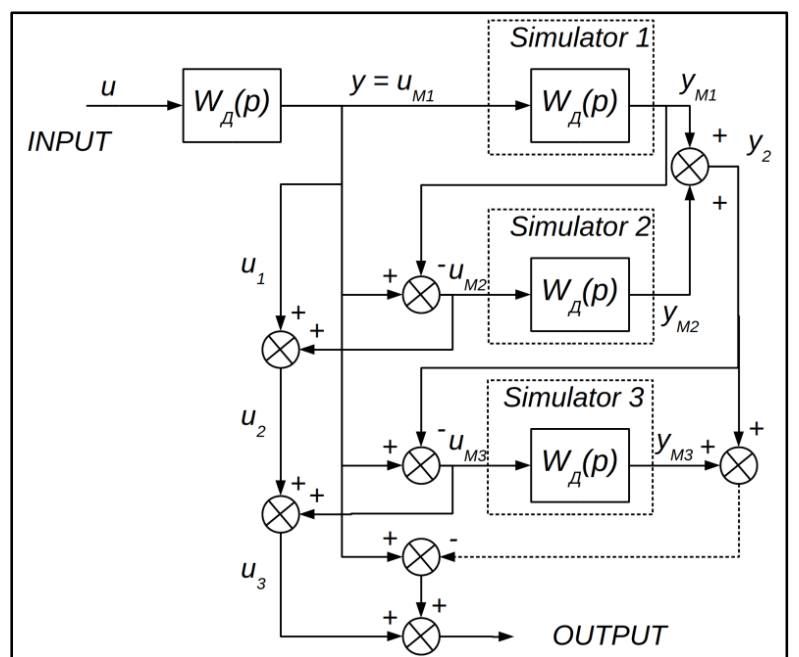

Figure 2. Structural scheme of the iterative measuring transducer.

$$
\left\{\begin{array}{c}
\dot{z}_{i}=A_{M i} z_{i}+B_{M i} u_{M i}, \\
y_{M i}=C_{M i} z_{i} .
\end{array}\right.
$$

The error identified by the previous iteration $u_{M i}=u_{1}-y_{M, i-1}$ becomes the input of the $i$-th simple transducer $u_{M i}, i=2,3 \ldots n$. Matrices $A_{M i}, B_{M i}, C_{M i}, i=1,2, \ldots N$ have the same meaning as the matrices $A, B, C$ in (1).

Therefore a mathematical model of the iterative MT can be represented by system

$$
\left\{\begin{aligned}
u_{M 1} & =u_{1}, \\
\dot{z}_{1} & =A_{M 1} z_{1}+B_{M 1} u_{M 1}, \\
y_{M 1} & =C_{M 1} z_{1}+D_{M 1} \eta_{M 1}, \\
u_{M 2} & =u_{1}-y_{M 1}, \\
\dot{z}_{2} & =A_{M 2} z_{2}+B_{M 2} u_{M 2}, \\
y_{M 2} & =C_{M 2} z_{2}+D_{M 2} \eta_{M 2}, \\
u_{M 3} & =u_{1}-y_{M 2}, \\
& \cdots \\
\dot{z}_{N} & =A_{M N} z_{N}+B_{M N} u_{M N}, \\
y_{M N} & =C_{M N} z_{N}+D_{M N} \eta_{M N}, \\
y_{1} & =u_{M 1}+u_{M 2}+\ldots+u_{M N}+u_{1}-y_{M 1}-y_{M 2}-\ldots-y_{M N} .
\end{aligned}\right.
$$

which reflects the idea of correction of dynamic error in the iterative principle, which consists in the sequential use of a number of simple MTs. The output of the system is presented by the sum of the observed signal and errors simulated by iterative chains.

We rewrite the mathematical model of the MT, which is represented by the scheme of the iterative MT, in the form of matrix equations for the case $N=2$. By introducing the notation

$x(t)=\left(z(t), u_{M}(t), y_{M}(t), y_{1}\right)^{T}, u(t)=\left(u_{1}, 0, \eta_{M 1}, \eta_{M 2}\right)^{T}$,

$y(t)=y_{1}(t)$.

(7) can be represented in the form of a Leontief type system

$\left\{\begin{aligned} L \dot{x} & =A x+B u, \\ y & =C x,\end{aligned}\right.$

where

$$
\begin{aligned}
L & =\left(\begin{array}{lllllll}
I & 0 & 0 & 0 & 0 & 0 & 0 \\
0 & I & 0 & 0 & 0 & 0 & 0 \\
0 & 0 & 0 & 0 & 0 & 0 & 0 \\
0 & 0 & 0 & 0 & 0 & 0 & 0 \\
0 & 0 & 0 & 0 & 0 & 0 & 0 \\
0 & 0 & 0 & 0 & 0 & 0 & 0 \\
0 & 0 & 0 & 0 & 0 & 0 & 0
\end{array}\right), \\
B & =\left(\begin{array}{lllll}
0 & 0 & 0 & 0 \\
0 & 0 & 0 & 0 \\
0 & 0 & D_{M 1} & 0 \\
0 & 0 & 0 & D_{M 2} \\
I & 0 & 0 & 0 \\
I & 0 & 0 & 0 \\
I & 0 & D_{1} & D_{2}
\end{array}\right), \quad C=(0,0,0,0,0,0, I)
\end{aligned}
$$

The use of Leontief type systems in modeling of the MT allows: 1) to model complex measuring devices with different types of connections between the transducers, considering the various types of feedback; 2) to solve not only the problem of restoration of dynamically distorted signals, but also problems connected with restoration of the system states.

\section{MATHEMATICAL MODEL OF OPTIMAL DYNAMIC MEASUREMENTS WITH INERTIA AND METHODS FOR ITS NUMERICAL STUDY SUBSECTIONS}

Considering the mathematical model of optimal dynamic measurements taking into account only inertia [14] of the MT, i.e. under the assumption of absence of disturbance or their insignificance, system (1), which models the MT, can be written in more simple way

$\left\{\begin{aligned} L \dot{x} & =A x+B u, \\ y & =C x .\end{aligned}\right.$

The initial condition (3) remains unchanged. By putting $y(t)=y(u, t)$ in the cost functional (5), we obtain

$J(u)=J_{1}(u)=\sum_{q=1}^{1} \int_{0}^{\tau}\left\|S y^{(q)}(u, t)-S y_{0}^{(q)}(t)\right\|^{2} d t$

where $y_{0}(t)=\left(y_{01}(t), y_{02}(t), \ldots, y_{o r}(t)\right)^{T}$ are real observations, $S y_{0}(t)$ are significant real observations, i.e. the ones that are used for searching the optimal measurement (though one can watch a greater number of characteristics of the process); $y(t)$ are virtual observations, obtained during mathematical modeling of the restoration of dynamic measurement; $S y(t)$ are significant virtual observations, i.e. the ones that are simulated by "the virtual MT", which are used for the comparison with significant real observations. We emphasize that the cost functional (9) minimizes the difference between the real and virtual observations, and the difference between the derivatives of these processes

$J(v)=\min _{u \in U_{\hat{o}}} J(u) \cdot$

An approximate solution to the optimal measurement problem is searched in the form of vector-polynomials

$u^{\ell}(t)=\left(\sum_{j=0}^{\ell} a_{1 j} t^{j}, \sum_{j=0}^{\ell} a_{2 j} t^{j}, \ldots, \sum_{j=0}^{\ell} a_{n j} t^{j}\right)^{T}$. 
The polynomials power is determined based on the requirements of numerical methods and calculation accuracy. In the numerical procedure [13] we search the coefficients of the vector-polynomials for which the cost functional is minimal. Note that the actual observation $y_{0}(t)$ was constructed by an interpolation procedure based on observed values $Y_{0 i}$ at the output of the real MT. The initial values of the coefficients vector of the polynomial (10) were taken equal to zero. Because of this, the search for optimal dynamic measurement with a given accuracy used to spend considerable time. We suggested the modification of the numerical method by introducing an additional procedure into the algorithm that uses linear splines [14]. As a result, the algorithm contains the following steps.

Step 1. Procedures of input and calculation of auxiliary parameters.

Step 2. Splitting $[0, \tau]$ into $n$ intervals. Consistent solving of the optimal measurement problem at each $i$-th interval $\left[\tau_{i-1}, \tau_{i}\right]$ (where $i=1, \ldots, n$ ) using linear functions for real observation $y_{0 i}(t)$, measurement $u_{i}(t)$ and optimal measurement $v_{i}(t)$.

As a result, on $[0, \tau]$ we get a piecewise linear function with the values $V_{0 i}=V_{0 i}\left(\tau_{i}\right)$ (Figure 3 ).

Step 3. Determination of the vector-function of real observations $y_{0}(t)$ using the values $Y_{0 i}$ and the interpolation procedure (Figure 4).

Step 4. Determination of the vector-function (10), whose

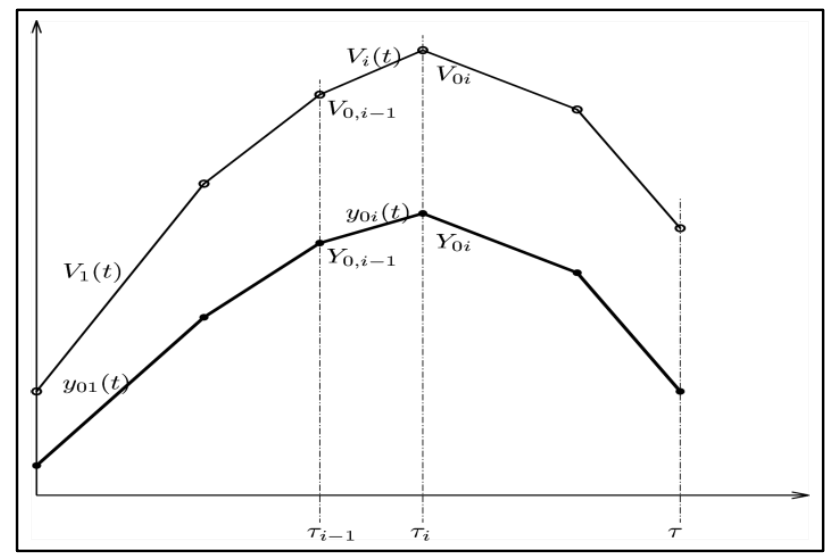

Figure 3. Step 2 of the proposed algorithm.

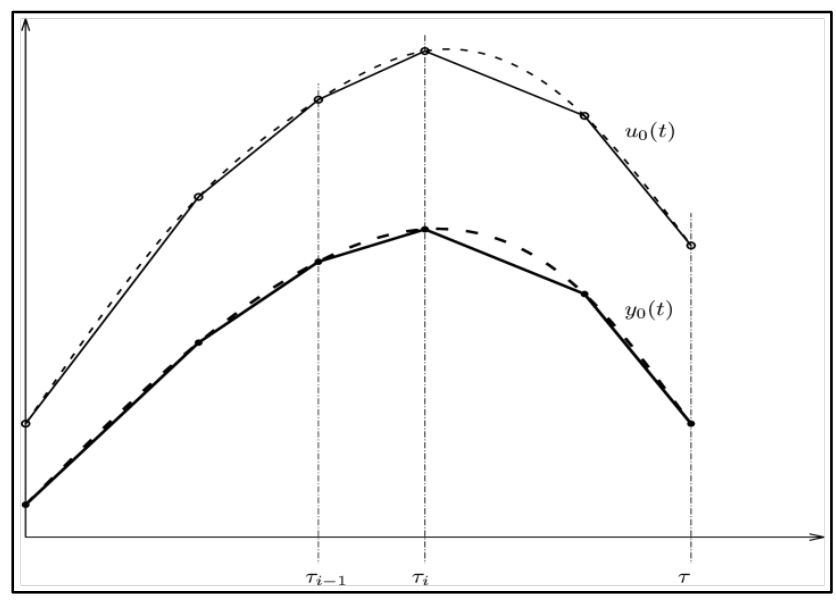

Figure 4 . Steps 3 and 4 of the proposed algorithm. coefficients will be the starting values for the numerical algorithm for the solution of the optimal measurement problem in the whole interval $[0, \tau]$ (Figure 4), using the values $V_{0 i}$ and the interpolation procedure.

Step 5. Search for virtual optimal measurement on the segment $[0, \tau]$. It uses the idea of the multistage alternatingvariable descent method with memory (Figure 5).

\section{MATHEMATICAL MODEL OF OPTIMAL DYNAMIC MEASUREMENTS WITH INERTIA AND RESONANCES ON THE OUTPUT OF THE MEASURING TRANSDUCER WITH DIFFERENT A PRIORI INFORMATION}

One of the most important problems in the theory of dynamic measurements is the restoration of the input signal distorted by deterministic disturbance, i.e. interference that does not change from experiment to experiment.

By deterministic we refer to disturbance caused by

- resonances in the MT, which lead, for example, to the blur of the lance-like input signals. Note that in this case it is assumed that frequencies of resonance disturbance are known;

- impacts of different nature, with a known form of useful signal, for example, a variable of voltage power sources.

We consider the modeling of optimal dynamic measurement under the assumption that the impact of deterministic disturbance is only on the output of the MT. In this case, the system modeling the MT has the form

$\left\{\begin{aligned} L \dot{x} & =A x+B u, \\ y & =C x+D \eta .\end{aligned}\right.$

The initial condition (3) remains unchanged. By putting $y(t)=y(u, \eta, t)$ in the cost functional (5), we obtain

$J(u)=J_{1}(u)=\sum_{q=1}^{1} \int_{0}^{\tau}\left\|S y^{(q)}(u, \eta, t)-S y_{0}^{(q)}(t)\right\|^{2} d t$

Note that the functionals (9) and (12) differ only by the functions of virtual observations, which are defined in systems (8) and (11) respectively. The virtual optimal measurement is determined by finding the minimum of the cost functional (12)

$J(v)=\min _{u \in U_{\partial}} J(u)$.

If the frequency of resonances in the output of the MT is known as a priori information then the function of real

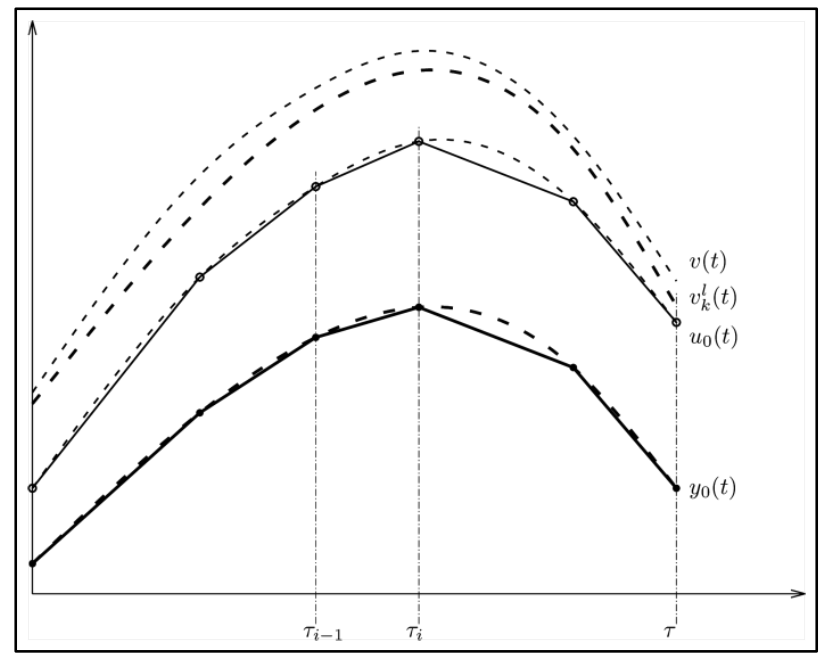

Figure 5 . Step 5 of the proposed algorithm. 
observations $y_{0}(t)$ is constructed by an interpolation of the observed values $Y_{0 i}$ at the output of the real MT.

If the form of the measurement function is known as a priori information, then the function of real observations $y_{0}(t)$ is constructed by an approximation procedure of the observed values $Y_{0 i}$ at the output of the real MT.

The method of numerical solution of the optimal measurement problem taking into account inertia and disturbance at the output of the MT in detail was described earlier. An approximate solution is sought in the form

$u^{\ell}(t)=\left(u_{1}^{\ell}, u_{2}^{\ell}, \ldots, u_{n}^{\ell}\right)^{T}=$

$=\left(\sum_{j=0}^{\ell} a_{1 j} \sin \varpi_{j} t, \sum_{j=0}^{\ell} a_{2 j} \sin \varpi_{j} t, \ldots, \sum_{j=0}^{\ell} a_{n j} \sin \varpi_{j} t\right)^{T}$.

The deterministic disturbance is considered as a vector function, each component of which is the sum of the resonances at known frequencies

$$
\eta(t)=\left(\sum_{j=0}^{M_{1}} a_{\omega_{1} j} \sin \omega_{1 j} t, \sum_{j=0}^{M_{2}} a_{\omega_{2} j} \sin \omega_{2 j} t, \ldots, \sum_{j=0}^{M_{m}} a_{\omega_{m} j} \sin \omega_{m j} t\right)^{T} .
$$

For the conduction of computational experiments, we construct a mathematical model of the MT, specified by a transfer function

$$
\begin{aligned}
& W_{g}(p)= \\
& =\frac{1}{\left(T_{1}^{2} p^{2}+2 \xi_{1} T_{1} p+1\right)\left(T_{2}^{2} p^{2}+2 \xi_{2} T_{2} p+1\right)\left(T_{3} p+1\right)\left(T_{4} p+1\right)}
\end{aligned}
$$

with known parameters $T_{1}=0,01 \mathrm{~s}, \xi_{1}=0,6, T_{2}=0,002 \mathrm{~s}$, $\xi_{2}=0,2, T_{3}=0,0005 \mathrm{~s}, T_{4}=0,0001 \mathrm{~s}$.

The mathematical model of the MT taking into account resonances has the form:

$$
\left\{\begin{array}{l}
\dot{x}_{1}=-x_{1}-0,9881 x_{2}+u, \\
\dot{x}_{2}=10000 x_{1}-119 x_{2}, \\
\dot{x}_{3}=x_{2}-x_{3}-0,999204 x_{4}, \\
\dot{x}_{4}=250000 x_{3}-119 x_{4}, \\
\dot{x}_{5}=2000 x_{4}-2000 x_{5}, \\
\dot{x}_{6}=10000 x_{5}-10000 x_{6}, \\
y=x_{6}+\eta .
\end{array}\right.
$$

For simplicity, B, C and D will be identity matrices. To evaluate the accuracy of the method, the found approximate optimal dynamic measurement is compared to the known input signal (precise dynamic measurement). Note that in real problems the precise dynamic measurement is not known.

Example 1. We consider the case when the resonance appears only at the output of the MT. Its frequency $\omega=5000$ is known, so $\eta=a_{\omega} \sin 5000 t$.

Figure 7 presents the real observation $y_{0}(t)$ found as a result of computational experiment, the optimal virtual measurement $v_{k}^{\ell}(t)$ and a precise dynamic measurement $v(t)$. In (13) frequencies $\varpi_{j}=6,25 j, j=0,1,2, \ldots \ell, \ell=32$, were taken, due to the amplitude-frequency properties of the transfer function of the MT (Figure 6).

As a result of computational experiment (Figure 7) the

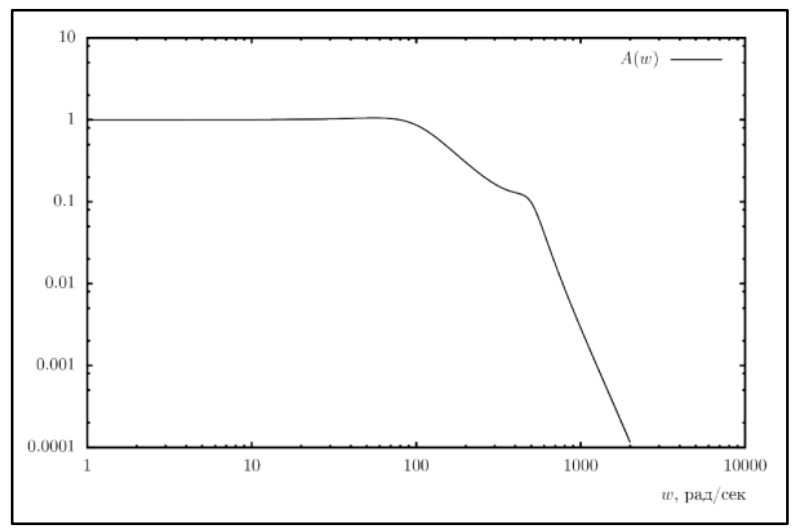

Figure 6. The amplitude-frequency properties of the transfer function of the MT

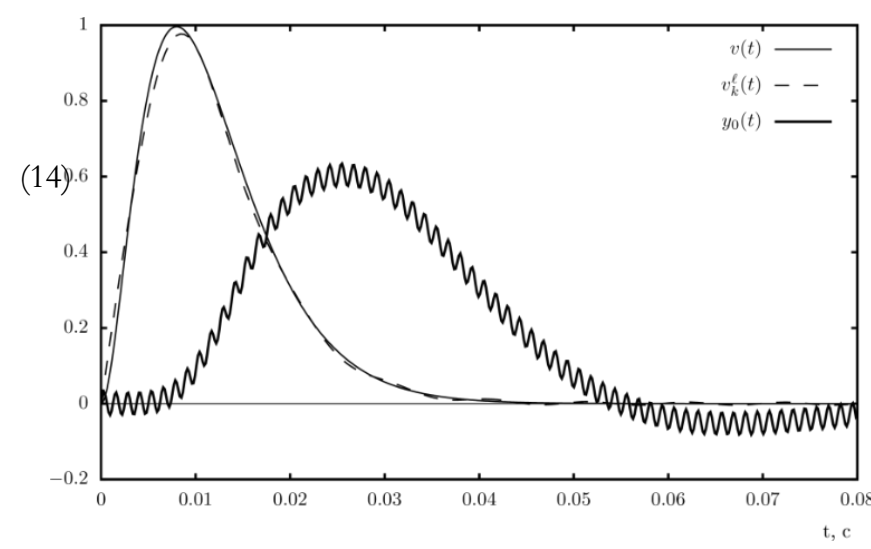

Figure 7. Results of computational experiment for the case of resonances at the output of the MT with known resonance frequency.

amplitude of the resonance disturbance $a_{\omega}=0,30151$ was found, so $\eta=0,30151 \sin 5000 t$.

A virtual optimal measurement as an approximate solution of the optimal dynamic measurement problem was obtained

$v_{k}^{\ell}=0,15638671 \sin (2 \pi \cdot 6,25 t)+0,24740745 \sin (2 \pi \cdot 12,5 t)+$

$+0,25821506 \sin (2 \pi \cdot 18,75 t)+0,21717253 \sin (2 \pi \cdot 25 t)+$ $+0,15941696 \sin (2 \pi \cdot 31,25 t)+0,10642029 \sin (2 \pi \cdot 37,5 t)+$ $+0,06576689 \sin (2 \pi \cdot 43,75 t)+0,03746629 \sin (2 \pi \cdot 50 t)+$ $+0,018902669 \sin (2 \pi \cdot 56,25 t)+0,00722448 \sin (2 \pi \cdot 62,5 t)$ $+0,00014199 \sin (2 \pi \cdot 68,75 t)-0,00397622 \sin (2 \pi \cdot 75 t)-$ $-0,00622392 \sin (2 \pi \cdot 81,25 t)-0,007312764 \sin (2 \pi \cdot 87,5 t)-$ $-0,00769625 \sin (2 \pi \cdot 93,75 t)-0,00766148 \sin (2 \pi \cdot 100 t)-$ $-0,00738759 \sin (2 \pi \cdot 106,25 t)-0,0069865873 \sin (2 \pi \cdot 112,5 t)-$ $-0,00652671 \sin (2 \pi \cdot 118,75 t)-0,00604986 \sin (2 \pi \cdot 125 t)-$ $-0,00558016 \sin (2 \pi \cdot 131,25 t)-0,00513176 \sin (2 \pi \cdot 137,5 t)-$ $-0,00471147 \sin (2 \pi \cdot 143,75 t)-0,00432264 \sin (2 \pi \cdot 150 t)-$ $-0,00396555 \sin (2 \pi \cdot 156,25 t)-0,00363959 \sin (2 \pi \cdot 162,5 t)-$ $-0,00334281 \sin (2 \pi \cdot 168,75 t)-0,00307338 \sin (2 \pi \cdot 175 t)-$ $-0,00282881 \sin (2 \pi \cdot 181,25 t)-0,002607121 \sin (2 \pi \cdot 187,5 t)-$ $0,00698658726010777 \sin (2 \pi \cdot 112,5 \mathrm{t})-$ $-0,0073875884086711 \sin (2 \pi \cdot 106,25 \mathrm{t})-$ $-0,00240593 \sin (2 \pi \cdot 193,75 t)-0,00222346 \sin (2 \pi \cdot 200 t)$. 
Example 2. We consider the case when the resonances appear at the output of the MT, their frequencies are not known, and the form of the signal $u(t)=a_{1} t^{2} e^{-a_{2} t}+a_{3}$ is known. Figure 8 presents the observed values $Y_{0 i}$ at the output of the the real MT, real observation $y_{0}(t)$ approximating these values, virtual optimal measurement $v_{k}^{\ell}(t)$ found as a result of the computational experiment and precise dynamic measurement $v(t)$.

As a result of the computational experiment an approximate solution of the optimal dynamic measurement problem $v_{k}^{\ell}(t)=115002,332 t^{2} e^{-250,24 t}$ was obtained.

\section{MATHEMATICAL MODEL OF OPTIMAL DYNAMIC MEASUREMENTS WITH INERTIA AND RESONANCES AT THE OUTPUT AND INPUT OF THE MEASURING TRANSDUCER}

We consider the model of an optimal dynamic measurement under the assumption on the impact of deterministic disturbances (resonances) both in the circuits of the MT and at the output of the MT. Note that it is assumed that the frequency of resonance disturbance is known. In this case, the system that models the MT has the form (1)

$$
\left\{\begin{array}{c}
L \dot{x}=A x+B u+G \varsigma, \\
y=C x+D \eta .
\end{array}\right.
$$

The initial condition (3) remains unchanged. The cost functional (5) has the form

$$
\begin{gathered}
J(u)= \\
=\sum_{q=1}^{1} \int_{0}^{\tau}\left\|S y^{(q)}(u, \varsigma, \eta, t)-\left(S y_{0}^{(q)}(u, \varsigma, \eta, t)-S y_{0}^{(q)}(\varsigma, \eta, t)\right)\right\|^{2} d t+ \\
+\sum_{q=1}^{1} \int_{0}^{\tau}\left\langle N_{q}(u+\varsigma)^{(q)}(t),(u+\varsigma)^{(q)}(t)\right\rangle d t
\end{gathered}
$$

where $\bar{y}_{0}(\varsigma, \eta, t)$ are real observations with zero input signal, $S \bar{y}_{0}(\varsigma, \eta, t)$ are those real observations with zero input signal that are used for restoration, $y_{0}(u, \varsigma, \eta, t)$ are real observations with measurement, $S y_{0}(u, \varsigma, \eta, t)$ are those real observations that are used for restoration of a dynamically distorted signal with nonzero input signal; $y(u, \varsigma, \eta, t)$ are virtual observatins obtained in mathematical modeling of dynamical measurement,

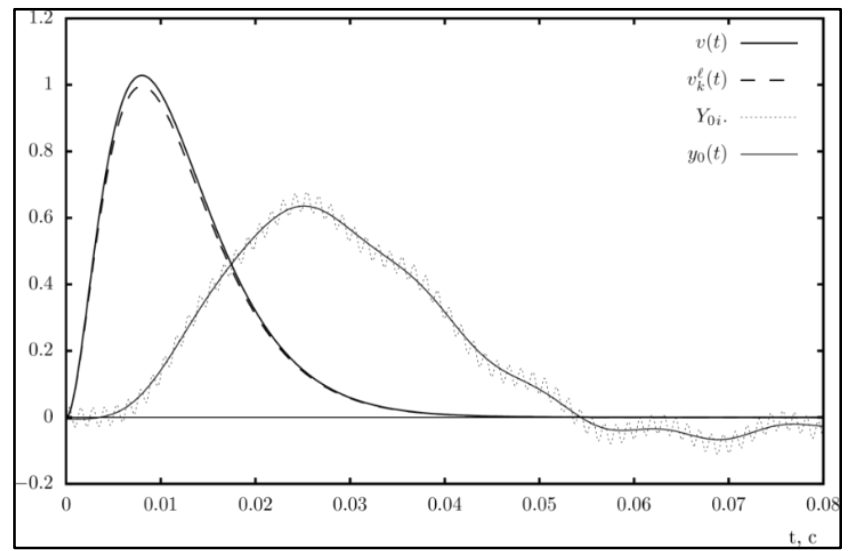

Figure 8. Results of computational experiment for the case of disturbances at the output of the MT with the known form of measured signal.
$S y(u, \varsigma, \eta, t)$ are those virtual observations that are used for restoration of a dynamically distorted signal in mathematical model. Virtual optimal measurement is determined by finding the minimum of the cost functional (16):

$J(v)=\min _{u \in U_{\curlywedge}} J(u)$.

Example 3. We consider the case when the frequencies of resonance at the output of the MT $\omega=5000$ and resonance in the circuits of the MT $\varphi=500$ are known, so $\eta=a_{\omega} \sin 5000 t$ and $\varsigma=a_{\varphi} \sin 500 t$. Figure 9 represents the real observations $\bar{y}_{0}(t)=\bar{y}_{0}(u, \varsigma, \eta, t)$ with zero input signal (this function is constructed by an interpolation procedure on the basis of observed values $\bar{Y}_{0 i}$ at the output of the real MT with zero values of measured signal).

The real observations are $y_{0}(t)=y_{0}(u, \varsigma, \eta, t)$ with nonzero input signal (this function is constructed by an interpolation procedure on the basis of observed values $Y_{0 i}$ at the output of the real MT with nonzero values of measured signal); the virtual optimal measurement $v_{k}^{\ell}(t)$ is found in the computational experiment; the precise dynamical measurement is $v(t)$.

As a result of computational experiment the approximate amplitudes of the resonances were found: $a_{\omega}=0,0300098$ and $a_{\varphi}=0,2999991, \quad$ so $\quad \eta=0,0300098 \sin 5000 t \quad$ and $\varsigma=0,2999991 \sin 500 t$.

\section{THE SET OF ADMISSIBLE MEASUREMENTS IN MATHEMATICAL MODELING OF DYNAMICAL MEASUREMENTS}

An essential part of the optimal measurement model is a set of admissible measurements $U_{\partial}$, which in fact is a compact convex subset in the space of observations $U$ and plays the same role as the set of admissible controls in optimal control theory:

$U_{\partial}=\left\{u \in U: \sum_{q=0}^{\theta} \int_{0}^{\tau}\left\|u^{(q)}(t)\right\|^{2} d t \leq d\right\}$,

where $\theta$ can take integer values from 0 to $p+1$. The integral form of the set of admissible measurements allows to use a priori information about the nature of the measured process in time. Note that the time integral of the square of the measured

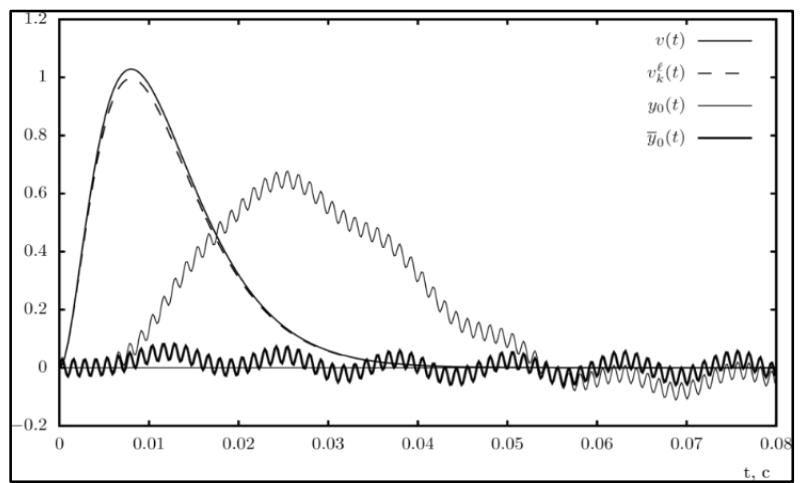

Figure 9. Results of computational experiment for the case of disturbances at the input and output of the MT with the known resonance frequences. 
process is the energy released during this process in the time interval $[0, \tau]$. This information can be obtained on the basis of laboratory tests, physical model, values of its parameters, etc. Moreover, this information may allow to allocate time intervals $\left[0, \tau_{1}\right],\left[0, \tau_{2}\right]\left[0, \tau_{2}\right], \ldots,[0, \tau] \quad\left(\right.$ or $\left[0, \tau_{1}\right],\left[\tau_{1}, \tau_{2}\right], \ldots$, $\left.\left[\tau_{i-1}, \tau_{i}\right], \ldots\right)$, reflecting several stages of the process, and to assess the set of admissible measurements at each of these intervals with constants $d_{1}, d_{2}, \ldots, d$, respectively. Note that the more accurate estimates are the more significant is the use of the set of admissible measurements at various stages of the measuring process. As a measure of accuracy of the mathematical model one can use the difference

$d-\tilde{d}=d-\sum_{q=0}^{\theta} \int_{0}^{\tau}\left\|v_{k}^{\ell(q)}(t)\right\|^{2} d t$

where $v_{k}^{\ell}(t)$ is an approximate virtual optimal measurement.

This approach was proposed to be used in a numerical algorithm to determine the stopping criteria of the algorithm, and selection of the sets of admissible measurements at different stages for more effective finding of an approximate solution [15].

We provide here an example about a combustion chamber: when comparing the speed and volume of delivery of the fuel mixture into the chamber, the calculated expected energy and burning rate, we can allocate time periods defining the stages of the combustion process, which will allow to estimate the value of energy released during these intervals. Data from laboratory tests, physical models of process, etc, can be used. In the combustion process two phases can be selected - transition or start of combustion and the operating mode of the mixture combustion in the combustion chamber. The transition process begins at the moment of ignition, when the contained working mixture is ignited. The operating mode of combustion starts with the moment when the volume of flammable mixture becomes constant in time (i.e. the pressure in the chamber becomes constant in time). Information about these phases allows to specify the set of admissible measurements, which will further improve the accuracy of virtual dynamic measurements.

We consider two cases of definition of the set of admissible measurements.

S1. The set of admissible measurements $U_{\partial}$ is given in the form (4) with $d=211,6097, \tau=0,08$ and $\theta=1$, i.e.

$U_{\partial}=\left\{u \in U: \sum_{q=0}^{1} \int_{0}^{0,08}\left\|u^{(q)}(t)\right\|^{2} d t \leq 211,6097\right\}$.

S2. The set of admissible measurements $U_{\partial}^{*}$ is given as follows:

$$
\begin{aligned}
& U_{\partial}^{*}=\left\{u \in U: u \in \bigcap_{i=1}^{3} U_{\partial i}\right\}, \\
& U_{\partial 1}=\left\{u \in U: \sum_{q=0}^{1} \int_{0}^{0,004}\left\|u^{(q)}(t)\right\|^{2} d t \leq 123,006\right\}, \\
& U_{\partial 2}=\left\{u \in U: \sum_{q=0}^{1} \int_{0,004}^{0,014}\left\|u^{(q)}(t)\right\|^{2} d t \leq 124,6636\right\},
\end{aligned}
$$

$U_{\partial 3}=\left\{u \in U: \sum_{q=0}^{1} \int_{0,014}^{0,08}\left\|u^{(q)}(t)\right\|^{2} d t \leq 38,9579\right\}$.

Figure 10 represents the solution of the optimal measurement problem in both cases S1 and S2: $v_{k}^{\ell}(t)$ and $v_{k}^{\ell *}(t)$ are the virtual optimal measurement for the case S1 and $\mathrm{S} 2$ respectively; $y_{0}(t)$ is the real observation.

\section{CONCLUSION}

This paper presents a number of mathematical models of optimal dynamic measurements with the inclusion of deterministic disturbances in various cases. The results of computational experiments show the adequacy of the mathematical models and the efficiency of computational methods.

In addition, we note that the theory of optimal dynamic measurements is developing in other directions, the results of which were not included in the paper:

- development of numerical methods using parallel computing;

- study of mathematical models of optimal dynamic measurements with a deterministic multiplicative effect on the state of the MT;

- study of mathematical models of optimal dynamic measurements with "noise" [16].

\section{REFERENCES}

[1] V.A. Granovsky, Dynamical Measurements: Fundamentals of Metrology Software (Energoizdat, Leningrad, 1984). (in Russian)

[2] A.L. Shestakov and G.A. Sviridyuk, A new approach to measurement of dynamically perturbed signals, Bulletin of the South Ural State University. Series: Mathematical Modelling, Programming and Computer Software 16 (192), 116 (2010). (in Russian)

[3] A.A. Belov and A.P. Kurdyukov, Descriptor Systems and Control Problems (FIZMATLIT, Moscow, 2015). (in Russian)

[4] K.H. Ruhm, Measurement Plus Observation - a Modern Structure of Metrology, in Measurement in Research and Industry World Congress International Measurement Confederation (XXI IMEKO), (Prague, Czechia, 2015).

[5] A. A. Kharkevich, Application of quadratic error criterion to estimate a linear distortion, Technical Physics 7 (5), 515 (1937). (in Russian)

[6] V.V. Solodovnikov, Introduction to the Statistical Dynamics of

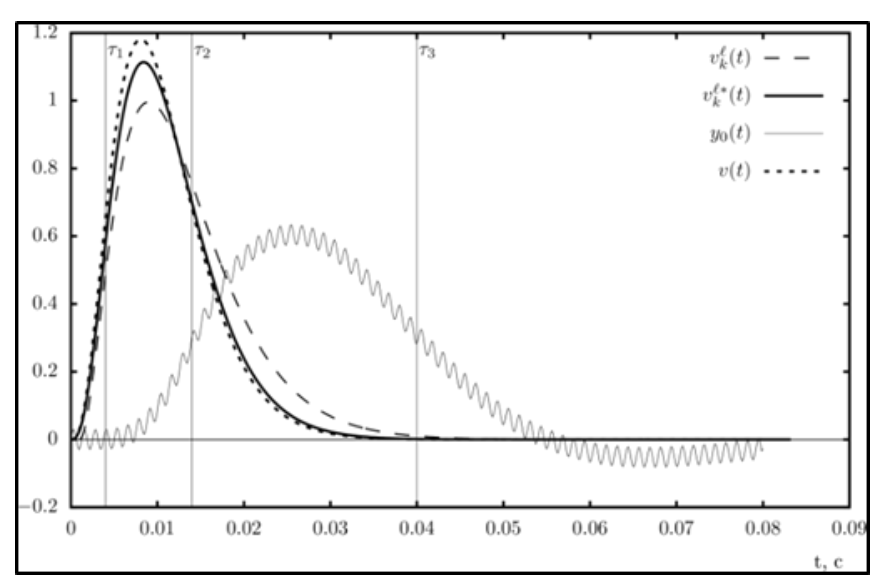

Figure 10. Results of computational experiment for different sets of admissible measurements. 
Remote Control Systems (Gostekhteorizdat, Moscow, Leningrad, 1952). (in Russian)

[7] Sh. Chang, Synthesis of Optimal Systems of Remote Control (Mashinostroenie, Moscow, 1964).

[8] D. Newton, L. Gould and L.D. Kaiser, Theory of Linear Tracking Systems (Fizmatgiz, Moscow, 1961).

[9] K. Merriam, Optimization. Theory and calculation of systems of feedback control (Mir, Moscow, 1967).

[10] A. Bryson and Ho Yu-Chi, Applied Optimal Control theory (translated from the edition of 1969, Mir, Moscow, 1972).

[11] Guang-Ren Duan. Analysis and Design of Descriptor Linear Systems, Advances in Mechanics and Mathematics 23 (Springer Science+Business Media, LLC, 2010)

[12] A. L. Shestakov, Measuring transducer of the dynamic parameters with the iterative principle for signal restoration,
Devices and systems. Management, control, diagnostics 10, 23 (1992).

[13] A.L. Shestakov, A.V. Keller and E.I. Nazarova, Numerical solution of the optimal measurement problem, Automation and Remote Control 1, 107 (2012).

[14] A.A. Ebel, On algorithm for numerical solution of optimal measurement problem using linear splines, Journal of Computational and Engineering Mathematics 3 (1), 37 (2016).

[15] Yu.V. Khudyakov, On adequacy of the mathematical model of the optimal dynamic measurement, Journal of Computational and Engineering Mathematics. 4 (2), 14 (2017).

[16] A.L. Shestakov, G.A. Sviridyuk, A.V. Keller and Yu.V. Khudyakov, The numerical algorithms for the measurement of the deterministic and stochastic signals, in Springer Proceedings in Mathematics \& Statistics, vol. 113 (2015), pp. 183-195. 